Mathematics

\section{What is the best way to lace your shoes?}

- he two most popular ways to lace shoes have historically been to use 'criss-cross' or 'straight' lacing — but are these the most efficient? Here we demonstrate mathematically that the shortest lacing is neither of these, but instead is a rarely used and unexpected type of lacing known as 'bowtie' lacing. However, the traditional favourite lacings are still the strongest.

The $2 n$ eyelets of an idealized shoe are the points of intersection of two vertical lines and $n$ equally spaced horizontal lines in the plane. The two columns of eyelets are one unit apart, and two adjacent rows of eyelets are a distance $h$ apart. An $n$-lacing of our shoe is a closed path in the plane that consists of $2 n$ line segments, the end points of which are the $2 n$ eyelets.

For any given eyelet, we require that at least one of the two segments that ends in it should not be contained in the same column as that eyelet; this condition ensures that every eyelet genuinely contributes towards pulling the two sides of the shoe together. Virtually all lacings that are actually used satisfy this condition.

We call a lacing 'dense' if neither of the

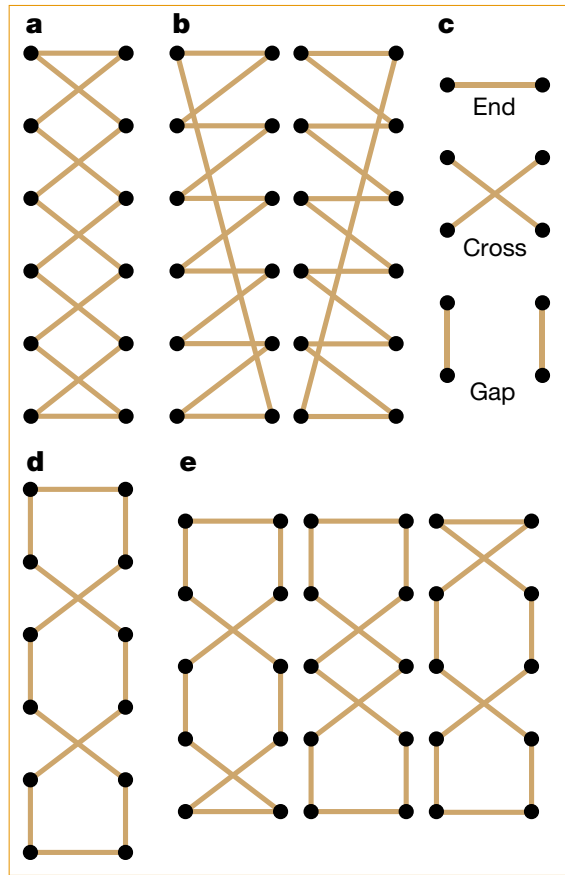

Figure 1 The strongest and the shortest shoe lacings. a, b, The most popular $n$-lacings, the criss-cross $n$-lacing (a) and the two straight $n$-lacings (b) are also the strongest $n$-lacings (here $n$ is the number of pairs of eyelets). $\mathbf{c - e}$, The shortest $n$-lacings are the bow-tie $n$-lacings. They are made up of ends, gaps and crosses (c). If $n$ is even, there is exactly one bow-tie $n$-lacing (d) consisting of the two ends at the top and bottom, $n / 2$ gaps and $n / 2-1$ crosses. If $n$ is odd, there are exactly $(n+1) / 2$ different bow-tie $n$-lacings (e) consisting of the two ends, $(n-1) / 2$ gaps and $(n-1) / 2$ crosses. two segments ending in any eyelet is contained in the same column as the eyelet - that is, a dense lacing zigzags back and forth between the two columns of eyelets as, for example, do the traditional lacings (Fig. $1 \mathrm{a}, \mathrm{b})$. Finally, we assume that $n$ is at least 2 .

Using standard combinatorial techniques, we find that the number of $n$-lacings is

$$
\frac{(n !)^{2}}{2} \sum_{k=0}^{m} \frac{1}{n-k}\left(\begin{array}{c}
n-k \\
k
\end{array}\right)^{2}
$$

where $m=n / 2$ for even $n$, and $m=(n-1) / 2$ for odd $n$. The number of dense $n$-lacings is

$$
\frac{n !(n-1) !}{2}
$$

The length of an $n$-lacing is the sum of the lengths of the segments that it consists of. Using the symmetries of the configuration of eyelets, it is possible to design a powerful list of local shortening rules and to use these to identify the bow-tie $n$-lacings as the shortest $n$-lacings (Fig. 1c-e). Furthermore, by generalizing earlier results ${ }^{1-4}$, we can show that the criss-cross $n$-lacing is the shortest dense $n$-lacing, even if the eyelets are not fully aligned. Note that it is also possible to identify the longest dense $n$-lacings for general $n$.

When you pull on the ends of a shoelace, it acts like a pulley. Ideally, the tension along the shoelace is a positive constant, $T$. This tension gives rise to a total tension, $T_{\text {hor }}$ of the pulley in the horizontal direction; that is, the direction in which the two sides of the shoe are being pulled together. This total tension, $T_{\text {hor }}$, is the sum of all horizontal components of $T$ along the different segments of the lacing. The strongest $n$-lacings are then $n$-lacings that maximize $T_{\text {hor }}$.

The unique dense 2-lacing is also the strongest 2-lacing. Note that the shortest $n$-lacing is independent of the distance $h$ between two adjacent rows of eyelets. In contrast, for $n>2$, the strongest $n$-lacing does depend on $h$. We can show that there is a positive value, $h_{n}$, such that the strongest $n$-lacings are: the criss-cross $n$-lacing, for $h<h_{n}$; the criss-cross $n$-lacing and the straight $n$-lacings, for $h=h_{n}$; and the straight $n$-lacings, for $h>h_{n}$.

For many real shoes with $n$ pairs of eyelets, the ratio of the distance between adjacent rows of eyelets and the distance between the columns is very close to $h_{n}$. This means that no matter whether you prefer to lace them straight or criss-crossed, you come close to maximizing the total horizontal tension when you pull on the two ends of one of your shoelaces.

And what is the strongest way to tie your shoelaces? ${ }^{5}$ Most people place one halfgranny knot on top of another (it is not essential to consider the loops here), which results in either a notoriously unstable granny knot or a very stable reef knot, depending on whether the two half-knots have the same or opposite orientation. As we have seen, hundreds of years of trial and error have led to the strongest way of lacing our shoes, but unfortunately the same cannot be said about the way in which most of us tie our shoelaces - with a granny knot.

Burkard Polster

School of Mathematical Sciences, PO Box 28M,

Monash University, Victoria 3800, Australia

e-mail: burkard.polster@sci.monash.edu.au

1. Halton, J. H. Math. Intell. 17, 37-41 (1995).

2. Misiurewicz, M. Math. Intell. 18, 32-34 (1996).

3. Stewart, I. Sci. Am. 78-80 (July 1996).

4. Stewart, I. Sci. Am. 86 (December 1996).

5. Smith, G. A. www.u.arizona.edu/ gasmith/knots/knots.html Competing financial interests: declared none.

COMMUNICATIONS ARISING Laser-Raman spectroscopy

\section{Images of the Earth's earliest fossils?}

ossil remains of the most ancient, minute forms of life on Earth and other planets are hard to recognize. Schopf et al. ${ }^{1}$ claim to have identified the biological remnant material known as kerogen in microscopic entities in rock by using Raman spectroscopic analysis. On the basis of a substantial body of published evidence, however, we contend that the Raman spectra of Schopf et al. ${ }^{1}$ indicate that these are disordered carbonaceous materials of indeterminate origin. We maintain that Raman spectroscopy cannot be used to identify microfossils unambiguously, although it is a useful technique for pinpointing promising microscopic entities for further investigation.

We believe that Schopf et al. have overinterpreted their Raman spectra in attributing biogenicity to the extremely ancient, fossil-like objects that they analysed, as already addressed by Brasier et al. ${ }^{2} \mathrm{We}$ disagree with the underlying assumption by Schopf et al. that Raman spectroscopy is sensitive to a distinctive carbon signal of organic matter (kerogen), and with their conclusion that "measurement of Raman point spectra (Fig. 3) [together with optical microscopy and Raman imaging] substantiates the biological origin of the oldest putative fossils".

Contrary to the inference by Schopf et al. ${ }^{1}$, laser-Raman microprobe spectroscopy does not reveal the chemical composition (as defined by geochemists) of a sample, but rather provides information about the molecular bonds of the constituent structural units. For instance, it can distinguish between carbon bonds in carbonate, in condensed benzene rings, in graphite and 
in diamond. It also characterizes the degree of crystalline order in solids. However, it does not provide a list of elemental components or even ratios of carbon, hydrogen and oxygen, which might help to establish the biogenicity of a compound.

Most pertinent to the analysis of putative microfossils is the fact that the Raman spectrum of carbonaceous (that is, carbon-dominated) materials is sensitive to the degree of ordering of the carbon they contain (distinguishing, for example, between amorphous carbon, poorly ordered graphitic material and highly crystalline graphite $)^{3-10}$. The Raman spectra of Schopf et al. ${ }^{1}$ confirm that their samples consist of highly disordered carbonaceous material and are consistent with the spectra of kerogens ${ }^{3,4}$. However, their spectra are indistinguishable from those of many other types of structurally disordered carbonaceous matter generated from a wide range of starting materials by a wide variety of processes. Those processes (including high-temperature heating of organic or inorganic compounds ${ }^{4-7}$, inorganic deposition from high-temperature synthetic fluids $^{3-5}$ and geological deposition from hydrothermal solutions ${ }^{8}$ ) and materials (for example, ion-bombarded graphite ${ }^{9}$ and graphite-intercalation compounds ${ }^{10}$ ) may be strictly non-biogenic. There are no distinctive features in the spectra shown by Schopf et al. ${ }^{1}$ that directly and unambiguously link them to kerogens.

Raman microprobe spectroscopy is useful for investigating the molecular structure of micrometre-sized features, such as putative microfossils, in rock. Showing that fossillike objects consist of highly disordered carbonaceous material by Raman spectroscopy provides necessary, but not sufficient, evidence that the objects are biogenic. Although the microscopic objects analysed by Schopf et al. ${ }^{1}$ may indeed be biogenic, we see nothing in their spectra that indicates the origin of their disordered carbonaceous material. The basic question remains unanswered: which measurable chemical and/or physical properties of a fossilized and/or altered material will unambiguously identify it as biological in origin?

\section{Jill Dill Pasteris, Brigitte Wopenka}

Department of Earth and Planetary Sciences,

Washington University, St Louis,

Missouri 63130-4899, USA

e-mail:pasteris@levee.wustl.edu

1. Schopf, J.W., Kudryavtsev, A. B., Agresti, D. G., Wdowiak, T. J. \& Czaja, A. D. Nature 416, 73-76 (2002).

2. Brasier, M. D. et al. Nature 416, 76-81 (2002).

3. Wopenka, B. \& Pasteris, J. D. Am. Mineral. 78, 533-557 (1993).

4. Beny-Bassez, C. \& Rouzaud, J. N. Scan. Electr. Microsc. 119-132 (1985)

5. Vidano, R. \& Fischbach, D. B. J. Am. Ceram. Soc. 61, 13-17 (1978).

6. Sato, Y., Kamo, M. \& Setaka, N. Carbon 16, 279-280 (1978)

7. Lespade, P., Al-Jishi, R. \& Dresselhaus, M. S. Carbon 20, 427-431 (1982)

8. Pasteris, J. D. in Applications of Microanalytical Techniques to Understanding Mineralizing Processes (eds McKibben, M. A.,
Shanks, W. C. \& Ridley, W. I.) 233-250 (Soc. Econ. Geol., Littleton, Colorado, 1998).

9. Elman, B. S., Shayegan, M., Dresselhaus, M. S., Mazurek, H. \& Dresselhaus, G. Phys. Rev. B 25, 4142-4156 (1982).

10. Dresselhaus, M. S. \& Dresselhaus, G. in Light Scattering in Solids III 2-57 (Springer, New York, 1982).

Schopf et al. reply - The criticism by Pasteris and Wopenka of our use of laser-Raman imagery to investigate the carbonaceous make-up of extremely ancient fossils ${ }^{1}$ focuses only on their Raman signature; however, our interpretation that the carbonaceous matter that makes up these specimens is biogenic is based on several lines of evidence, of which Raman spectroscopy is only one.

We did not state, nor did we imply, that Raman spectroscopic analysis can by itself be used to establish the biological origin of geochemically highly altered carbonaceous matter present in ancient sediments. We believe that the biogenicity of such matter, whether in fossil-like objects or sapropel-like detritus, should be demonstrated by a combination of data drawn from independent but mutually reinforcing lines of evidence.

For fossils in each of the four geological units we analysed ${ }^{1}$ - including those of the roughly 3,375-million-year (Myr)-old Kromberg Formation and 3,465-Myr-old Apex Chert, which are among the oldest fossils known - three lines of evidence are most compelling. These are their cellular morphology $y^{2,3}$, their carbonaceous molecularstructural make-up ${ }^{1-4}$, and the carbon isotope composition of such fossils ${ }^{5}$ and/or of co-existing particulate kerogen ${ }^{6,7}$, which have been shown by replicate analyses ${ }^{5-7}$ to be well within the range established for Precambrian biological organic matter on the basis of over 1,200 measurements from hundreds of fossilbearing units ${ }^{7}$.

Our study ${ }^{1}$, which focuses on the first two of these lines of evidence, is centred on the use of laser-Raman imagery (rather than on more conventional single-point measurements), a technique new to palaeobiology $y^{1,4}$. We showed that there is a oneto-one correlation of cellular morphology and carbonaceous make-up in individual microscopic fossils from each of the four units investigated. Our claim is that such an analysis based on a combination of morphology and chemistry together provides a powerful new means to investigate the biogenicity of putative fossil-like objects, a problem that for many decades has plagued the search for evidence of early life . $^{8}$

J. William Schopf ${ }^{\star}$, Anatoliy B. Kudryavtsev $\dagger$, David G. Agresti $\neq$, Thomas J. Wdowiak Andrew D. Czaja ${ }^{\star}$

${ }^{\star}$ Department of Earth \& Space Sciences, and Institute of Geophysics \& Planetary Physics, University of California, Los Angeles,

California 90095-1567, USA

e-mail: schopf@ess.ucla.edu $\dagger$ Department of Physics, and $\ddagger$ Astro and Solar System Physics Program, Department of Physics, University of Alabama, Birmingham,

Alabama 35294-1170, USA

1. Schopf, J. W., Kudryavtsev, A. B., Agresti, D. G., Wdowiak, T. J. \& Czaja, A. D. Nature 416, 73-76 (2002).

2. Schopf, J. W. in The Proterozoic Biosphere, a Multidisciplinary Study (eds Schopf, J. W. \& Klein, C.) 25-39, 1055-1117 (Cambridge Univ. Press, New York, 1992).

3. Schopf, J. W. Science 260, 640-646 (1993).

4. Kudryavtsev, A. B., Schopf, J. W., Agresti, D. G. \& Wdowiak, T. J. Proc. Natl Acad. Sci. USA 416, 73-76 (2002).

5. House, C. H. et al. Geology 28, 707-710 (2000).

6. Strauss, H. \& Moore, T. B. in The Proterozoic Biosphere, a Multidisciplinary Study (eds Schopf, J. W. \& Klein, C.) 709-798 (Cambridge Univ. Press, New York, 1992).

7. Strauss, H., Des Marais, D. J., Hayes, J. M. \& Summons, R. E. in The Proterozoic Biosphere, a Multidisciplinary Study (eds Schopf, J. W. \& Klein, C.) 117-127 (Cambridge Univ. Press, New York, 1992).

8. Schopf, J. W. \& Walter, M. R. in Earth's Earliest Biosphere, its Origin and Evolution (ed. Schopf, J. W.) 214-239 (Princeton Univ. Press, Princeton, New Jersey, 1983).

COMMUNICATIONS ARISING

\section{Palaeontology}

\section{Thermal alteration of the Earth's oldest fossils}

icroscopic carbonaceous structures found in ancient rocks could provide clues to early life on Earth if they turn out to be genuine fossil microorganisms. Here we show that thermal alteration of microbial remains embedded in a mineral matrix may significantly change their original morphology and produce structures that resemble those of what are claimed to be the Earth's oldest fossils'. These observations may shed light on the controversy ${ }^{2,3}$ that surrounds these microfossils from the 3,465-Myr-old Apex Chert of the early Archaean Warrawoona Group in northwestern Australia.

The biogenicity of these fossils has been called into question ${ }^{3}$ on the basis of suggestions that the Apex Chert structures were formed from amorphous graphite within multiple generations of metalliferous hydrothermal-vein chert and volcanic glass, and that the carbonaceous composition and characteristically biological carbon-isotope make-up of the carbonaceous filaments could have been products of non-biological (Fischer-Tropsch) organic synthesis ${ }^{3}$.

We have investigated structures that are present in silicified (chertified) cyanobacterial mats from the Bardo Mountains (Żdanów locality ${ }^{4}$ ) in southwestern Poland, which date to the early Silurian period (about 440 million years ago). The fossil mats occur in black, laminated radiolarian cherts, which have been interpreted as sediments that formed at moderate depths within the photic zone $e^{5}$. The mats are composed of cyanobacteria that are closely related to representatives of modern colonial chroococcaleans (particularly the 